\title{
Differential age susceptibility to influenza B/Victoria lineage viruses in the 2015 Australian influenza season
}

IG Barr ${ }^{1}$, D Vijaykrishna ${ }^{12}$, SG Sullivan ${ }^{1}$

1. WHO Collaborating Centre for Reference and Research on Influenza, VIDRL, Doherty Institute, Melbourne, Australia

2. Duke-NUS Medical School, Singapore

Correspondence: Ian G. Barr (Ian.Barr@influenzacentre.org)

Citation style for this article:

Barr I, Vijaykrishna D, Sullivan S. Differential age susceptibility to influenza B/Victoria lineage viruses in the 2015 Australian influenza season. Euro Surveill. 2016;21(4):pii=30118. DOI: http://dx.doi.org/10.2807/1560-7917.ES.2016.21.4.30118

Article submitted on 13 January 2016 / accepted on 26 January 2016 / published on 28 January 2016

Influenza B viruses make up an important part of the burden from seasonal influenza globally. The 2015 season in Australia saw an unusual predominance of influenza $B$ with a distinctive switch during the season from $\mathrm{B} /$ Yamagata/16/88 lineage viruses to $\mathrm{B} /$ Victoria/2/87 lineage viruses. We also noted significant differences in the age groups infected by the different $B$ lineages, with $\mathrm{B} /$ Victoria infecting a younger population than $B /$ Yamagata, that could not be explained by potential prior exposure.

The 2015 season was notable for the predominance of influenza B in Australia. According to the Australian Influenza Surveillance Report [1] for the period 1 January to 9 October, $61 \%$ of cases were typed as influenza $B$ and $38 \%$ influenza $A$ (29\% A (not subtyped), $7 \%$ $A\left(\mathrm{H}_{3} \mathrm{~N}_{2}\right)$ and $\left.2 \% \mathrm{~A}\left(\mathrm{H}_{1} \mathrm{~N}_{1}\right) \mathrm{pdmog}\right)$. That season was also interesting due to the waxing and waning of the two $B$ lineages over the season. Here, we summarise the lineage distribution using viruses submitted to the WHO Collaborating Centre for Reference and Research on Influenza in Melbourne for 2015 and compared these data with data from 2008, the last year when influenza $B$ viruses predominated in Australia.

\section{The 2015 influenza season in Australia}

Lineage data was available for 816 influenza B viruses from 2008 and 1,648 from 2015 that were received by the Centre from all over Australia. The formal representativeness of these samples is unknown. Generally there is a bias towards sampling from children and this is seen in most years in most general and sentinel surveillance systems and was also seen in the 2008 [2] and 2015 [1] Australian influenza seasons. It is unlikely that any bias would exist in selecting patients with a particular B lineage, and given the size and the geographical diversity of the samples tested, it is likely these data will provide an accurate estimate of the overall situation with influenza $B$ in Australia during these two years. During the 2015 pre-season period
(January-April) and the early part of the influenza season (May-June), B/Yamagata/16/88 lineage (B/Yam) viruses predominated. However, from July to November, $\mathrm{B} /$ Victoria/2/87 lineage (B/Vic) viruses increased rapidly and were dominant from August (Figure 1). Notably, this same switch was seen during the 2008 season with similar timing although almost no B/Vic lineage viruses were detected in Australia before June (Figure 1). The distributions of lineages during 2015 were similar when individual Australian states were examined, with the exception of the Northern Territory, which has a small population largely situated in the tropics, that had an almost total B/Vic year (36/39 B viruses). Australia's most populous state, New South Wales, experienced an increase in the proportion of B/ Vic viruses from low levels early in 2015 to $28 \%$ during the period from 15 June to 12 July 2015 [3] which according to our study continued to increase over the rest of the influenza season, and B/Vic viruses predominated from July onwards. Children and young adults carry a higher burden of influenza B disease than older adults and the elderly. According to the Australian Paediatric Surveillance Unit 2015 saw 88 children 15 years and younger (median: 3.3 years) hospitalised with severe complications of influenza between 1 July 2015 and 30 September 2015. Roughly two thirds $(n=59)$ were influenza B cases (lineage unknown) [1]. Overall, the average duration of hospitalisation was four days, 20 required an ICU admission, and there were three influenza-associated deaths, all associated with influenza $B$ infections [1].

\section{Antigenic and genetic drift}

The move in dominance by the B/Vic viruses in 2015 was not accompanied by any major antigenic changes from the $B / V i c$ viruses that circulated in smaller numbers in 2014. All Australian B/Vic viruses analysed by the Centre were antigenically B/Brisbane/60/2008like as they were in 2014 (data not shown). Equally, the 2015 B/Yam viruses that were analysed remained 


\section{FIGURE 1}

Relative frequency of influenza B subtypes received by month of specimen collection, Australia, $2008(\mathrm{n}=816)$ and $2015(\mathrm{n}=1,648)$

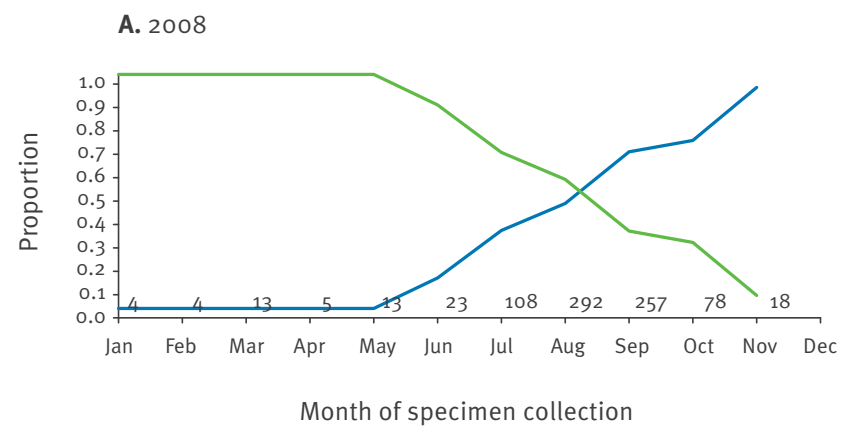

B. 2015

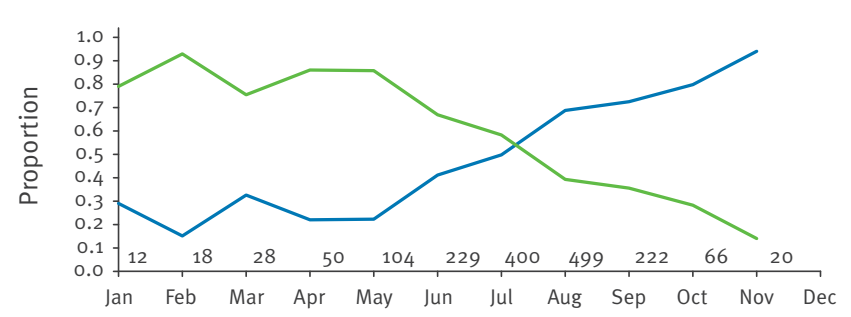

Month of specimen collection

$$
- \text { B/Vic - B/Yam }
$$

B/Vic: B/Victoria/2/87 lineage; B/Yam: B/Yamagata/16/88 lineage.

Numbers at the bottom of the figure are the total number of $B$ viruses tested for each month. Top panel: influenza $B$ subtypes in $2008(n=415$ B/Vic, $n=401$ B/Yam); bottom panel: influenza B subtypes in $2015(n=852$ B/Vic, $n=796$ B/Yam $)$.

antigenically B/Phuket3073/2013-like, similar to B/ Yam viruses that circulated in Australia from mid-2014 (data not shown). However, subtle phylogenetic differences in the haemagglutinin $(\mathrm{HA})$ genes of the two $B$ lineages were apparent (Figure 2). The HA gene phylogeny revealed a greater diversity for $\mathrm{B} /$ Yam viruses isolated in Australia during $2015(n=56)$ than during 2014 $(n=42)$ (Figure 2, top panel). The mean time to most recent common ancestor (mTMRCA) extended beyond 2012 and the isolates belonged to three antigenic types (B/Wisconsin/1/2010, B/Massachusetts/2/2012 and $B /$ Phuket/3073/2013), although the majority from 2015 (52 of the 56 viruses from 2015) belonged to the B/Phuket/3073/2013 clade (also known as group 3) with a mTMRCA in mid-2014. Reconstruction of nonsynonymous changes along this phylogeny revealed an amino acid substitution (M267V) in the subclade of B/Phuket/3073/2013-like viruses that were dominant during the 2015 season, suggesting that this mutation may have contributed to increased viral fitness. All Australian B/Vic viruses from $2015(n=54)$ were phylogenetically B/Brisbane/6o/2008-like (also referred to as clade $1 A$ ) with an mTMRCA in 2013. This clade was made up of three distinct subclades (Figure 2, bottom panel) the largest of which had the non-synonymous amino acid substitutions $V_{1} 61$ l and $I_{132} V$ that may have also enhanced the fitness of these viruses.

\section{Age distribution of influenza $B$ infections}

The age distribution of patients with confirmed B/ Vic infections in 2015 was positively skewed, with a greater number of infections among the younger age groups (mean: 26.4 years, median: 19.9 years). For B/ Yam infections, the age distribution was more even (mean: 42.4 years, median: 43.8 years; $p<0.001$ for Wilcoxon rank sum test). This age differential was less evident in 2008 where, despite the high proportion of viruses obtained from children younger than five years, the interquartile range indicated that $B / Y a m$ viruses affected a broader age range than the $B / V i c$ viruses (Figure 3). This age difference between lineages has previously been reported from a household study in Hong Kong [4]. There, children younger than 15 years had a 13-fold increased risk of secondary influenza infection with a B/Vic virus than with a B/Yam virus, during a period when both lineages were co-circulating. Similar findings were reported in population studies in southern China during the 2009 and 2010 seasons [5], over three seasons in Slovenia (2010-13 [6]) and in our earlier studies from eastern Australia and New Zealand, where major differences in lineage distribution were observed in subjects older than five years $[5,7]$.

\section{Discussion}

A predominance of influenza $B$ viruses in an influenza season occurs infrequently, usually in the order of once every 10 years. Prior to 2015 , it last occurred in Australia in the 2008 season, where $54 \%$ of typed viruses were influenza B, $43 \%$ were influenza $A$ and $3 \%$ untyped [2]. Similarly in Europe for the seasons from $2001 / 02$ to $2010 / 11$, influenza B was the majority influenza type (59.1\%) in only one season (2005/06). In the United States (US) over the same period, 2002/03 was the season with the highest proportion of influenza $B$ (42.6\%) among of all typed viruses [8]. The two antigenic and genetically distinct lineages of influenza $B$ viruses ( $B /$ Yam and $B /$ Vic) have co-circulated in various proportions since 2002 in most countries. Trivalent influenza vaccines (containing only one $B$ virus lineage) used over this time have tried to match these changing lineage circulation patterns. Because of a number of poor matches during the 2000s, quadrivalent vaccines (containing viruses from both $B$ lineages) were developed and have recently been introduced in order to improve vaccine effectiveness. The 2015 influenza vaccines licensed in Australia were all traditional inactivated virus vaccines (live attenuated influenza vaccines and recombinant vaccines were not available) with mostly trivalent vaccine containing only the $B /$ Yam component being used along with low levels of quadrivalent vaccine.

The reasons for the apparent differential age susceptibility between the two $B$ lineages described in this 


\section{FIGURE 2}

Maximum clade credibility trees showing the evolution of haemagglutinin genes of sequenced Australian influenza B viruses from 2014 and $2015(\mathrm{n}=168)$

A. Yamagata

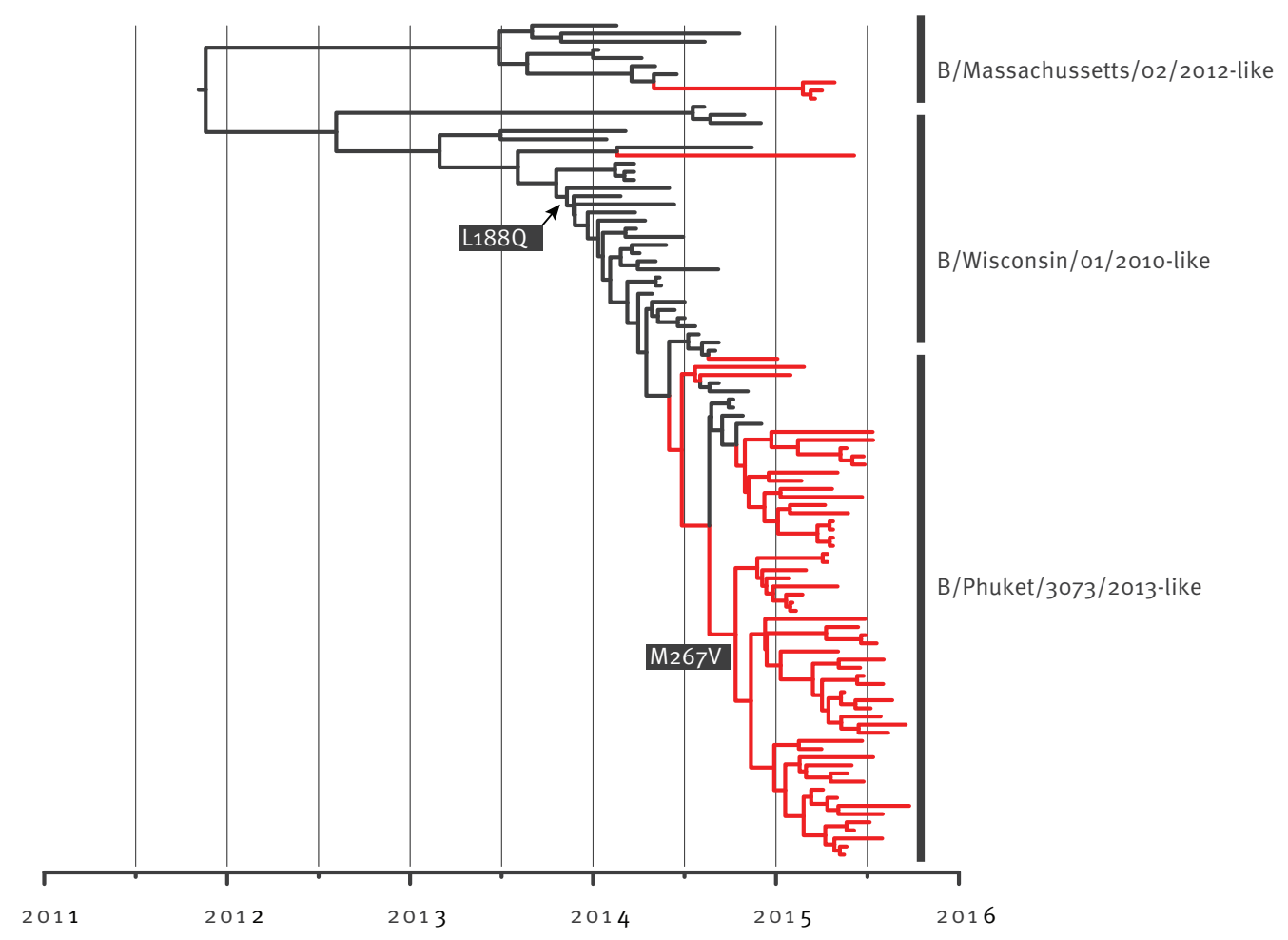

B. Victoria

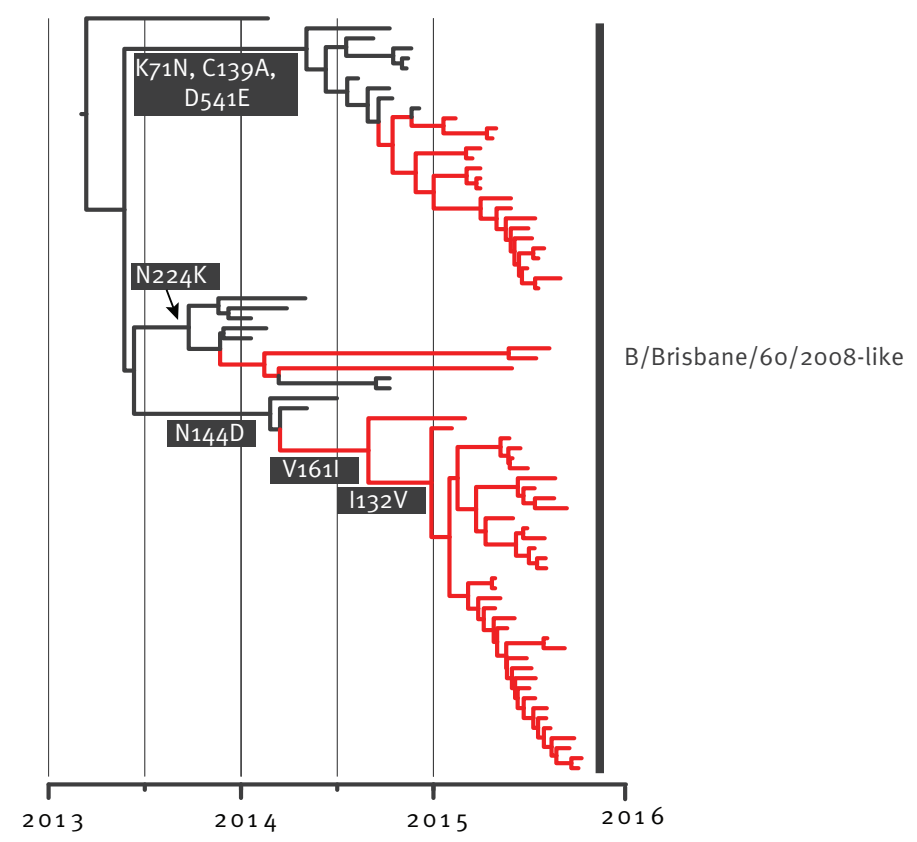

Phylogenies were inferred using a relaxed molecular clock model in a Bayesian Markov Chain Monte Carlo framework with the programme BEASTv1.8 [15].

Panel A: B/Yamagata/16/88 lineage viruses isolated in Australia in $2014(n=42)$ and $2015(n=56)$; Panel B: B/Victoria/2/87 lineage viruses isolated in Australia in $2014(n=16)$ and $2015(n=54)$. Red bars: Australian influenza B viruses from 2015; black bars: Australian influenza $B$ viruses from 2014. Non-synonymous amino acid changes that occurred during the evolution of the lineages are shown adjacent to the nodes. All available haemagglutinin (HA) sequences were obtained from The Global Initiative on Sharing All Influenza Data (GISAID; $\mathrm{http}: / /$ platform.gisaid.org/epiz/frontend). See the Table at the end of the article for details of the source and details of the virus and the sequencing laboratory. 
TABLE A

Australian influenza B viruses and haemagglutinin gene sequences used to construct Figure 2, obtained from The Global Initiative on Sharing All Influenza Data (GISAID) ${ }^{\mathrm{a}}$

\begin{tabular}{|c|c|c|c|c|c|}
\hline Segment ID & Collection date & Isolate name & Influenza B lineage & Originating laboratory & Authors ${ }^{b}$ \\
\hline $\mathrm{EPI}_{551283}$ & 2014-Aug-11 & B/Newcastle/21/2014 & Victoria & John Hunter Hospital & A \\
\hline $\mathrm{EPI}_{5} 61891$ & 2014-Dec-05 & B/Darwin/43/2014 & Victoria & Royal Darwin Hospital & A \\
\hline EPI541294 & 2014-Feb-18 & B/Tasmania/1/2014 & Victoria & Royal Hobart Hospital & A \\
\hline $\mathrm{EPI}_{541365}$ & 2014-Feb-21 & B/Perth/503/2014 & Victoria & Pathwest & A \\
\hline $\mathrm{EPI}_{529392}$ & 2014-Jan-20 & B/Brisbane/3/2014 & Victoria & QHSS & A \\
\hline EPI540771 & 2014-Jan-20 & B/Perth/501/2014 & Victoria & Pathwest & A \\
\hline $\mathrm{EPI}_{551321}$ & 2014-Jul-02 & B/South Australia/20/2014 & Victoria & IMVS & A \\
\hline EPI540747 & 2014-Mar-29 & B/Brisbane/12/2014 & Victoria & QHSS & A \\
\hline EPI551327 & 2014-May-03 & B/Sydney/19/2014 & Victoria & $\begin{array}{l}\text { Clinical Virology Unit, } \\
\text { CDIM }\end{array}$ & A \\
\hline EPI541291 & 2014-May-06 & B/Brisbane/13/2014 & Victoria & QHSS & A \\
\hline $\mathrm{EPI}_{562018}$ & 2014-Nov-14 & B/Brisbane/71/2014 & Victoria & QHSS & A \\
\hline $\mathrm{EPI}_{5} 61888$ & 2014-Nov-20 & B/Brisbane/74/2014 & Victoria & QHSS & A \\
\hline $\mathrm{EPI}_{5} 61873$ & 2014-0ct-09 & B/Brisbane/62/2014 & Victoria & QHSS & A \\
\hline $\mathrm{EPI}_{5} 61876$ & 2014-Oct-10 & B/Brisbane/63/2014 & Victoria & QHSS & A \\
\hline $\mathrm{EPI}_{5} 61924$ & $2014-0 c t-15$ & B/Victoria/7/2014 & Victoria & VIDRL & A \\
\hline $\mathrm{EPI}_{551336}$ & 2014-Sep-09 & B/Victoria/204/2014 & Victoria & Royal Chidrens Hospital & A \\
\hline EPI636426 & 2015-Apr-23 & $\mathrm{B} /$ Darwin/9/2015 & Victoria & Royal Darwin Hospital & B \\
\hline EPI636340 & 2015-Apr-28 & B/Brisbane/46/2015 & Victoria & QHSS & B \\
\hline EPI636409 & 2015-Apr-30 & B/Darwin/11/2015 & Victoria & Royal Darwin Hospital & $B$ \\
\hline EPI675691 & 2015-Aug-02 & B/Victoria/849/2015 & Victoria & Austin Health & B \\
\hline EPI675652 & 2015-Aug-03 & B/South Australia/1036/2015 & Victoria & IMVS & B \\
\hline EPI648854 & 2015-Aug-03 & B/Victoria/847/2015 & Victoria & Austin Health & B \\
\hline EPI675636 & 2015-Aug-04 & B/Newcastle/1012/2015 & Victoria & IMVS & $B$ \\
\hline EPI675677 & 2015-Aug-05 & $\mathrm{B} /$ Victoria/1009/2015 & Victoria & IMVS & $B$ \\
\hline EPI675663 & 2015-Aug-09 & B/Sydney/137/2015 & Victoria & Westmead Hospital & B \\
\hline EPI648856 & 2015-Aug-11 & B/Victoria/861/2015 & Victoria & Austin Health & B \\
\hline EPI675694 & 2015-Aug-18 & B/Victoria/898/2015 & Victoria & Austin Health & $B$ \\
\hline EPI675672 & 2015-Aug-20 & B/Tasmania/30/2015 & Victoria & Royal Hobart Hospital & $\mathrm{B}$ \\
\hline EPI675646 & 2015-Aug-30 & B/Perth/201/2015 & Victoria & Pathwest & B \\
\hline EPI630025 & 2015-Feb-05 & B/Brisbane/4/2015 & Victoria & QHSS & A \\
\hline EPI630050 & 2015-Feb-12 & B/South Australia/3/2015 & Victoria & IMVS & A \\
\hline EPI636504 & 2015-Jul-02 & B/South Australia/1015/2015 & Victoria & IMVS & B \\
\hline EPI648850 & 2015 -Jul-06 & B/Townsville/7/2015 & Victoria & QHSS & B \\
\hline EPI636421 & 2015-Jul-07 & B/Darwin/17/2015 & Victoria & Royal Darwin Hospital & $\mathrm{B}$ \\
\hline EPI648882 & 2015-Jul-12 & B/Victoria/524/2015 & Victoria & Monash Medical Centre & B \\
\hline EPI636621 & 2015-Jul-12 & B/Victoria/525/2015 & Victoria & Monash Medical Centre & $B$ \\
\hline EPI675604 & 2015-Jul-13 & $\mathrm{B} /$ Brisbane/186/2015 & Victoria & QHSS & $B$ \\
\hline EPI648868 & 2015-Jul-13 & B/Canberra/27/2015 & Victoria & Canberra Hospital & B \\
\hline EPI648846 & 2015-Jul-14 & B/Brisbane/185/2015 & Victoria & QHSS & B \\
\hline EPI675639 & 2015-Jul-15 & B/Newcastle/28/2015 & Victoria & John Hunter Hospital & B \\
\hline EPI648870 & 2015-Jul-19 & B/Canberra/29/2015 & Victoria & Canberra Hospital & $B$ \\
\hline EPI636388 & 2015-Jul-19 & B/Canberra/30/2015 & Victoria & Canberra Hospital & B \\
\hline EPI648848 & 2015-Jul-30 & B/Darwin/22/2015 & Victoria & Royal Darwin Hospital & B \\
\hline EPI675688 & 2015-Jul-31 & B/Victoria/843/2015 & Victoria & Austin Health & $B$ \\
\hline
\end{tabular}

IMVS: Institute of Medical and Veterinary Science; Pathwest: Pathwest QE II Medical Centre; QHSS: Queensland Health Scientific Services; VIDRL: Victoria Infectious Diseases Laboratory.

${ }^{a}$ All samples were sequenced and submitted by WHO Collaborating Centre for Reference and Research on Influenza, Melbourne, Australia, with the exception of $\mathrm{B} /$ Brisbane/47/2015 that was submitted by US Centers for Disease Control and Prevention.

${ }^{b}$ Authors: A: Deng Y-M, Iannello P, Spirason N, Jelley L, Lau H, Komadina N; B: Deng Y-M, lannello P, Spirason N, Lau H, Komadina N; C: Tilmanis D, Hurt A, Komadina N. 
TABLE B

Australian influenza B viruses and haemagglutinin gene sequences used to construct Figure 2, obtained from The Global Initiative on Sharing All Influenza Data (GISAID) ${ }^{\mathrm{a}}$

\begin{tabular}{|c|c|c|c|c|c|}
\hline Segment ID & Collection date & Isolate name & Influenza B lineage & Originating laboratory & Authors $^{b}$ \\
\hline EPI636549 & 2015-Jun-01 & B/Sydney/11/2015 & Victoria & $\begin{array}{l}\text { Clinical Virology Unit, } \\
\text { CDIM }\end{array}$ & B \\
\hline EPI636525 & 2015-Jun-04 & B/South Australia/49/2015 & Victoria & IMVS & B \\
\hline EPI636567 & 2015-Jun-15 & B/Tasmania/2/2015 & Victoria & Royal Hobart Hospital & B \\
\hline EPI636329 & 2015-Jun-27 & $\mathrm{B} /$ Brisbane/136/2015 & Victoria & QHSS & B \\
\hline EPI636415 & 2015-Jun-28 & B/Darwin/14/2015 & Victoria & Royal Darwin Hospital & B \\
\hline EPI636635 & 2015-Jun-28 & B/Victoria/557/2015 & Victoria & Monash Medical Centre & B \\
\hline EPI636577 & 2015-Jun-29 & B/Tasmania/5/2015 & Victoria & Royal Hobart Hospital & B \\
\hline EPI636465 & 2015-Jun-30 & B/Newcastle/1005/2015 & Victoria & IMVS & B \\
\hline EPI636560 & 2015-Mar-02 & B/Sydney/503/2015 & Victoria & Prince of Wales Hospital & B \\
\hline EPI636334 & 2015-Mar-16 & $\mathrm{B} /$ Brisbane/15/2015 & Victoria & QHSS & B \\
\hline EPI636584 & 2015-Mar-31 & B/Townsville/3/2015 & Victoria & QHSS & B \\
\hline EPI636605 & 2015-Mar-31 & B/Victoria/502/2015 & Victoria & Monash Medical Centre & B \\
\hline EPI636354 & 2015-May-08 & $\mathrm{B} /$ Brisbane/55/2015 & Victoria & QHSS & B \\
\hline EPI636361 & 2015-May-21 & B/Brisbane/69/2015 & Victoria & QHSS & B \\
\hline EPI636363 & 2015-May-24 & B/Brisbane/70/2015 & Victoria & QHSS & B \\
\hline EPI636369 & 2015-May-25 & B/Brisbane/73/2015 & Victoria & QHSS & B \\
\hline EPI636485 & 2015-May-28 & B/Perth/24/2015 & Victoria & Pathwest & B \\
\hline EPI636488 & 2015-May-28 & B/Perth/25/2015 & Victoria & Pathwest & B \\
\hline EPI636658 & 2015-May-30 & B/South Australia/48/2015 & Victoria & IMVS & B \\
\hline EPI636472 & 2015-May-31 & B/Newcastle/7/2015 & Victoria & John Hunter Hospital & B \\
\hline EPI675619 & 2015-Oct-03 & B/Darwin/65/2015 & Victoria & Royal Darwin Hospital & B \\
\hline EPI675622 & 2015-Oct-09 & B/Darwin/70/2015 & Victoria & Royal Darwin Hospital & B \\
\hline EPI675660 & 2015-Sep-07 & B/Sydney/1071/2015 & Victoria & IMVS & B \\
\hline EPI675655 & 2015-Sep-11 & B/South Australia/118/2015 & Victoria & IMVS & $\mathrm{B}$ \\
\hline EPI675602 & 2015-Sep-16 & B/Brisbane/1036/2015 & Victoria & IMVS & B \\
\hline EPI675686 & 2015-Sep-25 & B/Victoria/700/2015 & Victoria & Monash Medical Centre & B \\
\hline $\mathrm{EPI} 540782$ & 2014-Apr-04 & $\mathrm{B} /$ Newcastle/3/2014 & Yamagata & John Hunter Hospital & A \\
\hline EPI540744 & 2014-Apr-08 & $\mathrm{B} /$ Darwin/35/2014 & Yamagata & Royal Darwin Hospital & A \\
\hline $\mathrm{EPI} 540765$ & 2014-Apr-15 & B/Sydney/8/2014 & Yamagata & Prince of Wales Hospital & A \\
\hline EPI540762 & 2014-Apr-29 & B/Sydney/7/2014 & Yamagata & Prince of Wales Hospital & A \\
\hline EPI551286 & 2014-Aug-11 & B/Newcastle/22/2014 & Yamagata & John Hunter Hospital & A \\
\hline EPI551289 & 2014-Aug-12 & B/Newcastle/25/2014 & Yamagata & John Hunter Hospital & A \\
\hline $\mathrm{EPI}_{5} 61915$ & 2014-Dec-02 & B/Sydney/39/2014 & Yamagata & Westmead Hospital & A \\
\hline $\mathrm{EPI}_{5} 62030$ & 2014-Dec-03 & B/Perth/579/2014 & Yamagata & Pathwest & A \\
\hline $\mathrm{EPI}_{529622}$ & 2014-Feb-17 & B/Townsville/3/2014 & Yamagata & QHSS & A \\
\hline EPI540779 & $2014-F e b-25$ & B/Perth/505/2014 & Yamagata & Pathwest & $\mathrm{A}$ \\
\hline $\mathrm{EPI}_{529377}$ & 2014-Jan-12 & B/Darwin/4/2014 & Yamagata & Royal Darwin Hospital & A \\
\hline EPI529619 & 2014-Jan-28 & B/Brisbane/4/2014 & Yamagata & QHSS & A \\
\hline $\mathrm{EPI} 551274$ & 2014-Jul-03 & $\mathrm{B} /$ Newcastle/12/2014 & Yamagata & John Hunter Hospital & A \\
\hline EPI540912 & 2014-Jul-03 & B/South Australia/21/2014 & Yamagata & IMVS & A \\
\hline $\mathrm{EPI}_{551280}$ & 2014-Jul-24 & B/Newcastle/19/2014 & Yamagata & John Hunter Hospital & A \\
\hline EPI551324 & 2014-Jul-31 & B/Sydney/1002/2014 & Yamagata & IMVS & A \\
\hline EPI541331 & 2014-Jun-02 & B/Brisbane/22/2014 & Yamagata & QHSS & A \\
\hline EPI541279 & 2014-Jun-12 & B/South Australia/16/2014 & Yamagata & IMVS & A \\
\hline EPI541338 & 2014-Jun-13 & B/Newcastle/8/2014 & Yamagata & John Hunter Hospital & A \\
\hline
\end{tabular}

IMVS: Institute of Medical and Veterinary Science; Pathwest: Pathwest QE II Medical Centre; QHSS: Queensland Health Scientific Services; VIDRL: Victoria Infectious Diseases Laboratory.

${ }^{a}$ All samples were sequenced and submitted by WHO Collaborating Centre for Reference and Research on Influenza, Melbourne, Australia, with the exception of B/Brisbane/47/2015 that was submitted by US Centers for Disease Control and Prevention.

${ }^{b}$ Authors: A: Deng Y-M, Iannello P, Spirason N, Jelley L, Lau H, Komadina N; B: Deng Y-M, lannello P, Spirason N, Lau H, Komadina N; C: Tilmanis D, Hurt A, Komadina N. 
TABLE C

Australian influenza B viruses and haemagglutinin gene sequences used to construct Figure 2, obtained from The Global Initiative on Sharing All Influenza Data (GISAID) ${ }^{\mathrm{a}}$

\begin{tabular}{|c|c|c|c|c|c|}
\hline Segment ID & Collection date & Isolate name & Influenza B lineage & Originating laboratory & Authors $^{\mathrm{b}}$ \\
\hline $\mathrm{EPI}_{551277}$ & 2014-Jun-17 & B/Newcastle/17/2014 & Yamagata & John Hunter Hospital & A \\
\hline EPI540909 & 2014-Jun-30 & B/South Australia/1002/2014 & Yamagata & IMVS & A \\
\hline EPI541241 & 2014-Mar-07 & B/Brisbane/8/2014 & Yamagata & QHSS & A \\
\hline $\mathrm{EPI}_{551249}$ & 2014-Mar-24 & B/Brisbane/9/2014 & Yamagata & QHSS & A \\
\hline $\mathrm{EPI}_{540759}$ & 2014-Mar-26 & B/Sydney/5/2014 & Yamagata & Prince of Wales Hospital & A \\
\hline $\mathrm{EPI}_{540785}$ & 2014-Mar-28 & B/Newcastle/5/2014 & Yamagata & John Hunter Hospital & A \\
\hline EPI540906 & 2014-May-05 & B/Sydney/13/2014 & Yamagata & Westmead Hospital & A \\
\hline EPI540768 & 2014-May-06 & B/Sydney/9/2014 & Yamagata & Prince of Wales Hospital & A \\
\hline EPI540753 & 2014-May-14 & B/South Australia/5/2014 & Yamagata & IMVS & A \\
\hline EPI540756 & 2014-May-17 & B/South Australia/7/2014 & Yamagata & IMVS & A \\
\hline $\mathrm{EPI}_{541288}$ & 2014-May-27 & B/South Australia/1000/2014 & Yamagata & IMVS & $A$ \\
\hline $\mathrm{EPI}_{561918}$ & 2014-Nov-06 & $\mathrm{B} /$ Victoria/512/2014 & Yamagata & Monash Medical Centre & $A$ \\
\hline $\mathrm{EPI}_{5} 61885$ & 2014-Nov-11 & B/Brisbane/70/2014 & Yamagata & QHSS & A \\
\hline EPI630034 & 2014-Nov-14 & $\mathrm{B} /$ Canberra/20/2014 & Yamagata & Canberra Hospital & $A$ \\
\hline $\mathrm{EPI}_{5} 61870$ & 2014-Oct-08 & B/Brisbane/61/2014 & Yamagata & QHSS & $A$ \\
\hline $\mathrm{EPI}_{561912}$ & 2014-0ct-08 & B/Perth/569/2014 & Yamagata & Pathwest & A \\
\hline $\mathrm{EPI}_{561879}$ & 2014-Oct-20 & B/Brisbane/65/2014 & Yamagata & QHSS & A \\
\hline $\mathrm{EPI}_{5} 61882$ & 2014-Oct-27 & B/Brisbane/66/2014 & Yamagata & QHSS & A \\
\hline $\mathrm{EPI}_{561921}$ & $2014-0 c t-30$ & B/Victoria/6/2014 & Yamagata & VIDRL & A \\
\hline $\mathrm{EPI}_{551820}$ & 2014-Sep-02 & B/Victoria/804/2014 & Yamagata & Austin Health & A \\
\hline $\mathrm{EPI}_{551330}$ & 2014-Sep-08 & B/Townsville/1000/2014 & Yamagata & IMVS & $A$ \\
\hline $\mathrm{EPI}_{551264}$ & 2014-Sep-09 & B/Darwin/38/2014 & Yamagata & Royal Darwin Hospital & A \\
\hline $\mathrm{EPI}_{551333}$ & 2014-Sep-09 & B/Victoria/202/2014 & Yamagata & Royal Chidrens Hospital & $A$ \\
\hline EPI636392 & 2015-Apr-02 & B/Canberra/4/2015 & Yamagata & Canberra Hospital & B \\
\hline EPI636341 & 2015-Apr-03 & B/Brisbane/33/2015 & Yamagata & QHSS & $\mathrm{B}$ \\
\hline EPI630067 & 2015-Apr-05 & B/Victoria/500/2015 & Yamagata & Monash Medical Centre & A \\
\hline EPI636553 & 2015-Apr-09 & B/Sydney/5/2015 & Yamagata & $\begin{array}{l}\text { Clinical Virology Unit, } \\
\text { CDIM }\end{array}$ & B \\
\hline EPI642630 & 2015-Apr-14 & B/Brisbane/47/2015 & Yamagata & WHO CC & NA \\
\hline EPI636506 & 2015-Apr-23 & B/South Australia/12/2015 & Yamagata & IMVS & $B$ \\
\hline EPI636606 & 2015-Apr-25 & B/Victoria/503/2015 & Yamagata & Monash Medical Centre & B \\
\hline EPI648860 & 2015-Apr-25 & B/Victoria/530/2015 & Yamagata & Monash Medical Centre & $B$ \\
\hline EPI636345 & 2015-Apr-28 & $\mathrm{B} /$ Brisbane/5o/2015 & Yamagata & QHSS & $B$ \\
\hline EPI648852 & 2015-Aug-01 & B/Victoria/845/2015 & Yamagata & Austin Health & $B$ \\
\hline EPI675669 & 2015-Aug-02 & B/Sydney/70/2015 & Yamagata & Westmead Hospital & $B$ \\
\hline EPI675644 & 2015-Aug-03 & B/Perth/166/2015 & Yamagata & Pathwest & B \\
\hline EPI675657 & 2015-Aug-04 & B/Sydney/1031/2015 & Yamagata & IMVS & $B$ \\
\hline EPI675666 & 2015-Aug-13 & B/Sydney/153/2015 & Yamagata & Westmead Hospital & $\mathrm{B}$ \\
\hline EPI675675 & 2015-Aug-21 & B/Tasmania/32/2015 & Yamagata & Royal Hobart Hospital & $\mathrm{B}$ \\
\hline EPI630031 & 2015-Feb-03 & B/Canberra/1/2015 & Yamagata & Canberra Hospital & $A$ \\
\hline EPI630047 & 2015-Feb-10 & B/South Australia/2/2015 & Yamagata & IMVS & C \\
\hline EPI630053 & 2015 -Feb-21 & B/South Australia/4/2015 & Yamagata & IMVS & A \\
\hline EPI630055 & 2015 -Feb-23 & B/South Australia/5/2015 & Yamagata & IMVS & $\mathrm{C}$ \\
\hline EPI630064 & 2015 -Feb-26 & B/Townsville/1/2015 & Yamagata & QHSS & A \\
\hline EPI630016 & 2015-Jan-04 & $\mathrm{B} /$ Brisbane/1/2015 & Yamagata & QHSS & A \\
\hline
\end{tabular}

IMVS: Institute of Medical and Veterinary Science; Pathwest: Pathwest QE II Medical Centre; QHSS: Queensland Health Scientific Services; VIDRL: Victoria Infectious Diseases Laboratory.

a All samples were sequenced and submitted by WHO Collaborating Centre for Reference and Research on Influenza, Melbourne, Australia, with the exception of B/Brisbane/47/2015 that was submitted by US Centers for Disease Control and Prevention.

${ }^{b}$ Authors: A: Deng Y-M, Iannello P, Spirason N, Jelley L, Lau H, Komadina N; B: Deng Y-M, Iannello P, Spirason N, Lau H, Komadina N; C: Tilmanis D, Hurt A, Komadina N. 
TABLE D

Australian influenza B viruses and haemagglutinin gene sequences used to construct Figure 2, obtained from The Global Initiative on Sharing All Influenza Data (GISAID) ${ }^{a}$

\begin{tabular}{|c|c|c|c|c|c|}
\hline Segment ID & Collection date & Isolate name & Influenza B lineage & Originating laboratory & Authors ${ }^{b}$ \\
\hline EPI630061 & 2015-Jan-28 & B/Sydney/2/2015 & Yamagata & $\begin{array}{c}\text { Clinical Virology Unit, } \\
\text { CDIM }\end{array}$ & $A$ \\
\hline EPI630058 & $2015-\operatorname{Jan}-30$ & B/Sydney/100o/2015 & Yamagata & IMVS & A \\
\hline EPI636636 & 2015-Jul-07 & B/Victoria/543/2015 & Yamagata & Monash Medical Centre & $B$ \\
\hline EPI636618 & 2015-Jul-09 & B/Victoria/519/2015 & Yamagata & Monash Medical Centre & $\mathrm{B}$ \\
\hline EPI636601 & 2015-Jul-13 & $\mathrm{B} /$ Victoria/32/2015 & Yamagata & VIDRL & $B$ \\
\hline EPI636387 & 2015-Jul-14 & B/Canberra/28/2015 & Yamagata & Canberra Hospital & $B$ \\
\hline EPI636627 & 2015-Jul-14 & B/Victoria/532/2015 & Yamagata & Monash Medical Centre & $B$ \\
\hline EPI636641 & 2015-Jul-21 & B/Victoria/952/2015 & Yamagata & Royal Chidrens Hospital & B \\
\hline EPI675641 & 2015-Jul-29 & B/Perth/136/2015 & Yamagata & Pathwest & $B$ \\
\hline EPI636592 & 2015-Jun-01 & B/Victoria/301/2015 & Yamagata & Melbourne Pathology & $B$ \\
\hline EPI636531 & 2015-Jun-06 & B/South Australia/50/2015 & Yamagata & IMVS & $\mathrm{B}$ \\
\hline EPI636566 & 2015-Jun-06 & B/Tasmania/1/2015 & Yamagata & Royal Hobart Hospital & B \\
\hline EPI636313 & 2015-Jun-14 & B/Brisbane/100/2015 & Yamagata & QHSS & $B$ \\
\hline EPI636322 & 2015-Jun-18 & $\mathrm{B} /$ Brisbane/118/2015 & Yamagata & QHSS & $\mathrm{B}$ \\
\hline EPI636460 & 2015-Jun-22 & B/Newcastle/1003/2015 & Yamagata & IMVS & $B$ \\
\hline EPI636468 & 2015-Jun-22 & B/Newcastle/20/2015 & Yamagata & John Hunter Hospital & $B$ \\
\hline EPI636326 & 2015-Jun-25 & B/Brisbane/132/2015 & Yamagata & QHSS & B \\
\hline EPI636379 & 2015-Jun-25 & B/Canberra/13/2015 & Yamagata & Canberra Hospital & $B$ \\
\hline EPI636535 & 2015-Jun-25 & B/South Australia/71/2015 & Yamagata & IMVS & $B$ \\
\hline EPI636574 & 2015-Jun-27 & B/Tasmania/4/2015 & Yamagata & Royal Hobart Hospital & $\mathrm{B}$ \\
\hline EPI636383 & 2015-Jun-28 & B/Canberra/15/2015 & Yamagata & Canberra Hospital & B \\
\hline EPI636541 & 2015-Jun-29 & B/Sydney/1013/2015 & Yamagata & IMVS & $B$ \\
\hline EPI630019 & 2015-Mar-02 & B/Brisbane/11/2015 & Yamagata & QHSS & A \\
\hline EPI630022 & 2015-Mar-20 & B/Brisbane/19/2015 & Yamagata & QHSS & A \\
\hline EPI636455 & 2015-May-03 & B/Newcastle/1/2015 & Yamagata & John Hunter Hospital & $\mathrm{B}$ \\
\hline EPI636349 & 2015-May-04 & $\mathrm{B} /$ Brisbane/54/2015 & Yamagata & QHSS & B \\
\hline EPI636515 & 2015-May-04 & B/South Australia/22/2015 & Yamagata & IMVS & $\mathrm{B}$ \\
\hline EPI636514 & 2015-May-05 & B/South Australia/18/2015 & Yamagata & IMVS & B \\
\hline EPI636500 & 2015-May-17 & B/South Australia/1000/2015 & Yamagata & IMVS & $\mathrm{B}$ \\
\hline EPI636611 & 2015-May-17 & B/Victoria/507/2015 & Yamagata & Monash Medical Centre & $\mathrm{B}$ \\
\hline EPI636482 & 2015-May-22 & B/Perth/21/2015 & Yamagata & Pathwest & $B$ \\
\hline EPI636521 & 2015-May-22 & B/South Australia/28/2015 & Yamagata & IMVS & $\mathrm{B}$ \\
\hline EPI636593 & 2015-May-25 & B/Townsville/6/2015 & Yamagata & QHSS & $\mathrm{B}$ \\
\hline EPI675616 & 2015-Sep-17 & B/Darwin/61/2015 & Yamagata & Royal Darwin Hospital & B \\
\hline EPI675683 & 2015-Sep-24 & $\mathrm{B} /$ Victoria/698/2015 & Yamagata & Monash Medical Centre & $B$ \\
\hline
\end{tabular}

IMVS: Institute of Medical and Veterinary Science; Pathwest: Pathwest QE II Medical Centre; QHSS: Queensland Health Scientific Services; VIDRL: Victoria Infectious Diseases Laboratory.

a All samples were sequenced and submitted by WHO Collaborating Centre for Reference and Research on Influenza, Melbourne, Australia, with the exception of B/Brisbane/47/2015 that was submitted by US Centers for Disease Control and Prevention.

${ }^{b}$ Authors: A: Deng Y-M, Iannello P, Spirason N, Jelley L, Lau H, Komadina N; B: Deng Y-M, Iannello P, Spirason N, Lau H, Komadina N; C: Tilmanis D, Hurt A, Komadina N. 
FIGURE 3

Age distribution of confirmed influenza B cases by lineage, Australia, $2008(n=780)$ and $2015(n=1,638)$
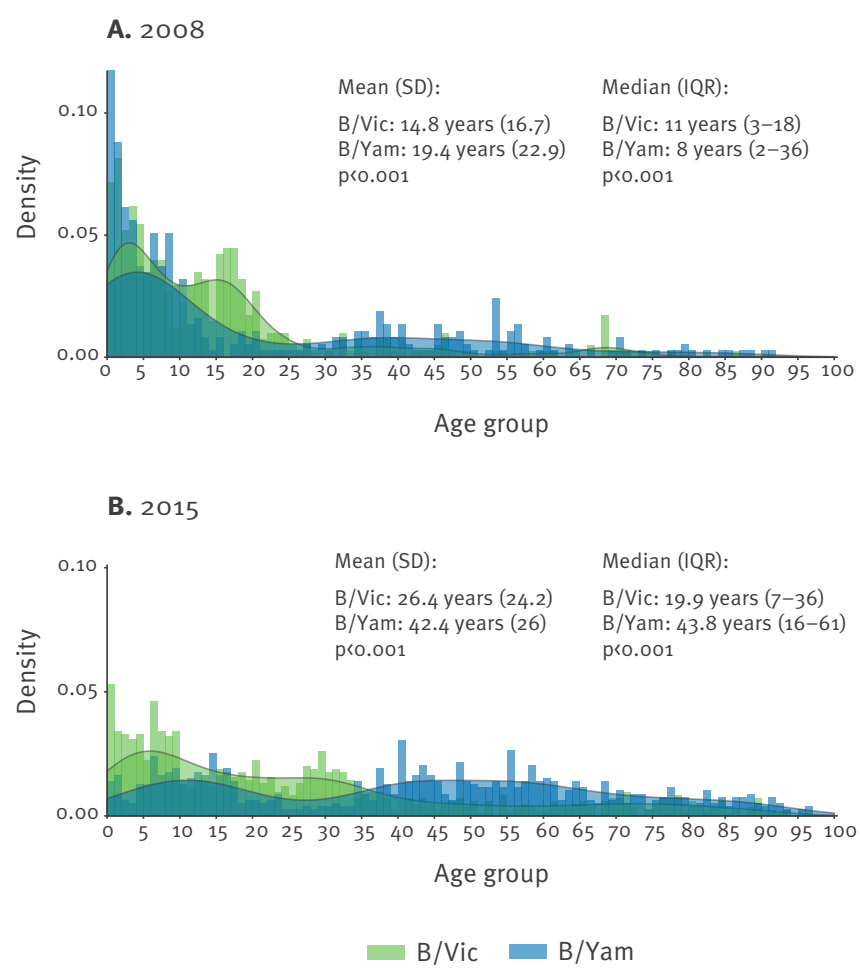

IQR: interquartile range; SD: standard deviation.

Data shown are samples for which age data was available. Bars show the histogram in one-year age increments by lineage. The shaded areas indicate the smoothed density estimate of the age distribution. Values provided on the graphs for each lineage are mean (SD) or median (IQR). P values are for the t-test comparing mean age between the two lineages and the Wilcoxon rank-sum test for comparing medians.

study are unknown. It is, however, well known that the different influenza types/subtypes do affect different age profiles; both seasonal and 2009 pandemic $A\left(\mathrm{H}_{1} \mathrm{~N}_{1}\right)$ as well as influenza $B$ viruses infect a younger population than $A\left(\mathrm{H}_{3} \mathrm{~N}_{2}\right)$ viruses [9], although in recent years, the median age of influenza $A\left(\mathrm{H}_{1} \mathrm{~N}_{1}\right)$ pdmog cases has been increasing $[6,10]$, again for unknown reasons. Studies to date have not shown differences in clinical presentation for the different B lineages [4,5], but long-term data on hospitalisations and deaths are lacking. In a study by Paddock et al. on deaths attributed to confirmed influenza $B$ in the US from 2000 to 2010 , the majority of subjects were 18 years and younger (34/45 cases), and a slightly higher proportion of infections were B/Vic compared with B/Yam ( 25 vs 17 deaths, respectively, in those cases that could be characterised) [11]. More studies are required to determine if there is indeed any difference in outcomes following severe infections with either of the $B$ lineages in different age groups.

It is probable that the prior exposure history of the different age groups has influenced our observations. However, this is difficult to deduce from the present data. For example, five-year-old Australian children in 2015 were likely to have been exposed to a mixture of B/Yam viruses, which circulated in 2013 and 2014, and B/Vic viruses, which circulated from 2009 to 2012, as was the case in for five-year-olds in 2008. Possible exposure therefore fails to explain the elevated proportion of five year-old children infected with $\mathrm{B} /$ Yam viruses in 2008 or with B/Vic in 2015. In addition, it is unlikely given the low levels of childhood vaccination in Australia that this this would have significantly altered the circulation patterns of the influenza B lineages. Vaccination uptake is generally below $10 \%$ among Australian children [12]. Childhood influenza vaccination is only recommended for children of aboriginal descent five years and younger and for children six months and older with comorbidities [13].

We have suggested previously that there may be some fundamental differences in the receptor specificity of the different influenza $B$ lineages and that the distribution or density of receptors for influenza $B$ viruses in the respiratory tract of humans may differ with age [7]. Others have shown differential responses of children to B/Yam and B/Vic antigens contained in influenza vaccines that might also contribute to differential susceptibility to these two lineages 14]. Further work is needed to fully understand the basis of these observations and to determine if the differences are due to receptor variation or density during ageing or prior exposure history or a mixture of both. If indeed young children are at an elevated risk of infection with $B /$ Vic viruses, then it may be prudent to prioritise distribution of quadrivalent vaccines (containing viruses from both $B$ lineages) to this age group. This is relevant to the current northern hemisphere influenza season where the trivalent vaccine contains a B/Yam lineage virus, but $\mathrm{B} / \mathrm{Vic}$ lineage viruses are in our view likely to increase substantially during the current season. Use of the quadrivalent vaccine for this subgroup (or preferably for the whole population) would be potentially advantageous in improving influenza vaccine effectiveness.

\section{Acknowledgements}

The Melbourne WHO Collaborating Centre for Reference and Research on Influenza is supported by the Australian Government Department of Health. The authors would like to thank all laboratories that supplied samples used in these studies, including the Australian WHO National Influenza Centres at VIDRL in Victoria, ICPMR in Sydney, Pathwest in Perth and other laboratories and hospitals in Australia. DV is supported by the Duke-NUS Signature Research Program funded by the Agency of Science, Technology and Research, Singapore and the Ministry of Health Singapore, and by contract HHSN272201400006C from the National Institute of Allergy and Infectious Disease, National Institutes of Health, Department of Health and Human Services, United States.

We acknowledge the authors, originating and submitting laboratories of the sequences from GISAID's EpiFlu Database on which this research is based (see Table). All submitters of data may be contacted directly via the GISAID website www. gisaid.org 


\section{Conflict of interest}

None declared.

\section{Authors' contributions}

IB and SS wrote the manuscript, SS performed the epidemiological analysis, DV performed phylogenetic analyses, all authors revised the manuscript.

\section{References}

1. Australian Government Department of Health. Australian Influenza Surveillance Report. No.10, 2015, reporting period: 26 September to 9 October 2015. Canberra: Australian Government Department of Health; 2015. Available from: http://www.health.gov.au/internet/main/publishing.nsf/ Content/cda-surveil-ozflu-flucurr.htm/\$File/AustralianInfluenza-Surveillance-Report.pdf

2. Kaczmarek M, Owen R, Barr IG. Annual report of the National Influenza Surveillance Scheme, 2008.Commun Dis Intell Q Rep. 2010;34(1):8-22.PMID: 20521494

3. Jennings Z, Carter I, McPhie K, Kok J, Dwyer DE. Increased prevalence of influenza B/Victoria lineage viruses during early stages of the 2015 influenza season in New South Wales, Australia: implications for vaccination and planning. Euro Surveill. 2015;20(31):21201. DOI: 10.2807/1560-7917. ES2015.20.31.21201 PMID: 26290426

4. Xu C, Chan KH, Tsang TK, Fang VJ, Fung RO, Ip DK, et al. Comparative Epidemiology of Influenza B Yamagata- and Victoria-Lineage Viruses in Households. Am J Epidemiol. 2015;182(8):705-13. DOI: 10.1093/aje/kwV110 PMID: 26400854

5. Tan Y, Guan W, Lam TT, Pan S, Wu S, Zhan Y, et al. Differing epidemiological dynamics of influenza B virus lineages in Guangzhou, southern China, 2009-2010. JVirol. 2013;87(22):12447-56. DOI: 10.1128/JVI.01039-13 PMID: 24027322

6. Sočan M, Prosenc K, Učakar V, Berginc N. A comparison of the demographic and clinical characteristics of laboratoryconfirmed influenza B Yamagata and Victoria lineage infection.J Clin Virol. 2014;61(1):156-60. DOI: 10.1016/j. jcv.2014.06.018 PMID: 25034374

7. Vijaykrishna D, Holmes EC, Joseph U, Fourment M, Su YC, Halpin R, et al. The contrasting phylodynamics of human influenza B viruses. eLife. 2015;4:e05055. DOI: 10.7554/ eLife.05055 PMID: 25594904

8. Ambrose CS, Levin MJ. The rationale for quadrivalent influenza vaccines.Hum Vaccin Immunother. 2012;8(1):81-8. DOI: 10.4161/hv.8.1.17623 PMID: 22252006

9. Kelly HA, Grant KA, Williams S, Fielding J, Smith D. Epidemiological characteristics of pandemic influenza H1N1 2009 and seasonal influenza infection. Med J Aust. 2009;191(3):146-9.PMID: 19645642

10. Skowronski DM, Chambers C, Sabaiduc S, Janjua NZ, Li $G$, Petric $M$, et al. Pre- and postpandemic estimates of 2009 pandemic influenza $A\left(\mathrm{H}_{1} \mathrm{~N}_{1}\right)$ seroprotection to inform surveillance-based incidence, by age, during the 2013-2014 epidemic in Canada. J Infect Dis. 2015;211(1):109-14. DOI: 10.1093/infdis/jiu366 PMID: 24973459

11. Paddock CD, Liu L, Denison AM, Bartlett JH, Holman RC, Deleon-Carnes $\mathrm{M}$, et al. Myocardial injury and bacterial pneumonia contribute to the pathogenesis of fatal influenza $B$ virus infection. J Infect Dis. 2012;205(6):895-905. DOI: 10.1093/ infdis/jir861 PMID: 22291193

12. Sullivan SG, Chilver MB, Higgins G, Cheng AC, Stocks NP. Influenza vaccine effectiveness in Australia: results from the Australian Sentinel Practices Research Network. Med J Aust. 2014;201(2):109-11. DOI: 10.5694/mja14.00106 PMID: 25045991

13. Australian Government Department of Health and Ageing. Part 4 Vaccine-preventable diseases. The Australian Immunisation Handbook. 10th ed. Canberra: National Health and Medical Research Council; 2015. p. 251-6. Available from: http://www. immunise.health.gov.au/internet/immunise/publishing.nsf/ Content/immunise-influenza (Accessed 20 Jan. 2016)

14. Skowronski DM, Hottes TS, De Serres G, Ward BJ, Janjua NZ, Sabaiduc S, et al. Influenza B/Victoria antigen induces strong

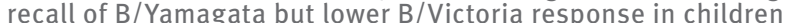
primed with two doses of B/Yamagata. Pediatr Infect Dis J. 2011;30(10):833-9. DOI: 10.1097/INF.ob013e31822db4dc PMID: 21857263
15. Drummond AJ, Suchard MA, Xie D, Rambaut A. Bayesian phylogenetics with BEAUti and the BEAST 1.7. Mol Biol Evol. 2012;29(8):1969-73. DOI: 10.1093/molbev/mss075 PMID: 22367748

\section{License and copyright}

This is an open-access article distributed under the terms of the Creative Commons Attribution (CC BY 4.0) Licence. You may share and adapt the material, but must give appropriate credit to the source, provide a link to the licence, and indicate if changes were made.

This article is copyright of the authors, 2016. 